Rev SINAPSIS, Vol. 6, Nº 1, Junio 2015

ISSN $1390-7832$

\title{
El aula virtual como herramienta de enseñanza - aprendizaje en bachillerato
}

\section{La importancia del aula virtual en el bachillerato}

\author{
Génessis Lisbeth Laz Rodrígez ${ }^{(1)}$ \\ Rosa Lilibeth Laz Rodríguez ${ }^{(2)}$ \\ (1) Unidad Educativa ITSUP \\ (2) Unidad Educativa ITSUP
}

\author{
Contacto: glisbeth_05@hotmail.com
}

Receptado: 20/02/2015 Aceptado: 11/04/2015

\section{Resumen}

El artículo como parte de un proyecto de investigación, aborda la necesidad de la utilización del aula virtual con fines educativos por parte de los estudiantes de bachillerato en Ecuador, se plantea como objetivo el análisis de la utilización del aula virtual y su incidencia en el aprendizaje de los estudiantes. Para la puesta en marcha de esta experiencia se encuestaron 16 profesores, 110 estudiantes y 2 directivos de la Unidad Educativa ITSUP; a través de los resultados arrojados se concluyó que los docentes y estudiantes limitan la utilización del aula virtual en actividades tradicionales como el envío de tareas, desaprovechando de esta manera la gama de actividades que el aula virtual contiene y que permiten junto a otras herramientas la adquisición de verdaderos aprendizajes.

Palabras clave: Actividades, docentes, tecnología, recurso didáctico

\section{The virtual classroom as a teaching tool in the High school}

\begin{abstract}
The article as part of a research project states the need for the use of the virtual classroom for educational purposes by High school students in Ecuador. The objective was to analyze the use of the virtual classroom and its impact on the learning of the students. For the implementation of this experience 16 teachers, 110 students and 2 directors of "Unidada Educativa ITSUP" were surveyed. The results concluded that teachers and students limit the use of virtual classroom in
\end{abstract}


Rev SINAPSIS, Vol. 6, № 1, Junio 2015

traditional activities such as homework and the like, thus wasting the range of activities that contains the virtual classroom and allowing other tools for acquisition of the real learning.

Keywords: Activities, teaching, technology, teaching resource

\section{Introducción}

Ante los avances de la ciencia y la tecnología, las instituciones educativas empiezan a tomar conciencia sobre la necesidad de crear nuevas políticas y estrategias orientadas a reducir la brecha entre el currículum, la investigación y la práctica pedagógica, las mismas que permitan responder con pertinencia a las exigencias de la sociedad actual mediante el desarrollo de capacidades y destrezas para enfrentar con éxito la era de la información.

Los educadores han encontrado un mundo de posibilidades al desarrollo de su práctica docente a través de integrar las nuevas tecnologías como un recurso más en el proceso de enseñanzaaprendizaje, que les ha permitido promover y facilitar la actitud participativa y creadora de los alumnos, la enseñanza individualizada del aprendizaje interactivo, la formación a distancia y de nuevas metodologías como la enseñanza apoyada por computadora, lo que ocasiona una verdadera transformación en el proceso de enseñanza aprendizaje al ceder el papel protagónico al alumno. El planteamiento de actividades de enseñanza y aprendizaje virtual ha de seguir los criterios de la enseñanza adaptativa en tanto que aporten elementos de flexibilización tanto a los alumnos como a los profesores. (Barbera E. y., S/F)

Un factor importante a considerar en la formación de los estudiantes es el aprendizaje, aspecto que en los actuales momentos se considera en relación a la utilización de la tecnología aplicada a la educación; es decir, de manera concreta al aula virtual, que es utilizada como recurso de apoyo educativo. El aula virtual no debe ser solo un mecanismo para la distribución de la información, sino que debe ser un sistema donde las actividades involucradas en el proceso de aprendizaje puedan tomar lugar, es decir que deben permitir interactividad, comunicación, aplicación de los conocimientos, evaluación y manejo de la clase.

Por otra parte, las funciones del docente cambian cuando debe desarrollar sus actividades en un entorno virtual de enseñanza -aprendizaje, que además deja de tener limitaciones geográficas, 
Rev SINAPSIS, Vol. 6, Nº 1, Junio 2015

físicas, temporales y que tiende a dar respuesta a grupos de alumnos cada vez más heterogéneos y diversos (en el sentido más extenso de estas dos palabras), y por ello creemos que debe redefinirse su tarea profesional así como las funciones que deberá asumir en el desarrollo de ésta (Gisbert, $\mathrm{S} / \mathrm{F})$.

En concordancia a lo anteriormente mencionado, la Unidad Educativa ITSUP, tiene como misión la formación integral de los estudiantes, pero una formación de avanzada de acuerdo a los estándares educativos de calidad; por lo que, como parte de esa formación los estudiantes complementariamente a las tareas y actividades presenciales, deben realizar tareas virtuales en cada una de las asignaturas, contando con los recursos físicos, humanos y tecnológicos necesarios para el desarrollo de las mismas; sin embargo, a medida que los estudiantes avanzan en edad y nivel de estudios, se evidencia una escasa utilización del aula virtual con fines educativos, ya que las nuevas generaciones, también llamados nativos digitales, utilizan estos medios para otros fines, como la ampliación de sus redes sociales, dejando en un segundo plano el aprovechamiento de estos recursos para investigar, reforzar o ampliar los aprendizajes adquiridos, lo que sin lugar a dudas incide en el aprendizaje de los mismos, considerando que periódicamente los docentes requieren trabajos asignados en este espacio.

Teniendo en cuenta estas dificultades, este trabajo se encuentra fundamentado hacia el objetivo de analizar la utilización del aula virtual y su incidencia en el aprendizaje de los estudiantes como parte de la educación integral.

\section{Materiales y métodos}

Para la realización de este trabajo se utilizó el apoyo documental, donde se consultaron diferentes publicaciones realizadas por estudiosos del tema y que sirvieron de base para la fundamentación teórica de la investigación. Se realizaron además encuestas a directivos, docentes y estudiantes, material que propició la validación de los resultados.

\section{Desarrollo}

Un elemento imprescindible en el nuevo modelo educativo integrando las nuevas tecnologías es el aula virtual, concepto que se ha venido desarrollando a partir de la década de los ochenta, término 
Rev SINAPSIS, Vol. 6, № 1, Junio 2015

ISSN $1390-7832$

que se le adjudica a Roxanne Hiltz quien la define como el empleo de comunicaciones mediadas por computadoras para crear un ambiente electrónico semejante a las formas de comunicación que normalmente se producen en el aula convencional.

En la literatura consultada existe una gran amplitud de autores y organizaciones que han trabajado el término aula virtual, así: (Passey, 2000, pág. 23) manifiesta que "el aula virtual es la integración organizada de muchos recursos digitales de texto, imagen, sonido y animación”, los mismos que se convierten en estímulos que capturan la atención, despiertan el interés e invitan al trabajo de quienes la utilizan en este caso específico de los adolescentes, que por haber nacido en la era tecnológica se los considera como nativos digitales, por lo que poseen ciertas habilidades innatas.

Por su parte, (Harasim, 2000, pág. 54) considera que "El aula virtual es el medio en el cual los educadores y educandos se encuentran para realizar actividades que conducen al aprendizaje" es decir que adicionalmente las clases presenciales el docente continua desarrollando su labor educativa con cada uno de sus estudiantes a través de este recurso digital, consiguiendo sin lugar a dudas las metas planteadas. También el aula virtual, está concebida como un Sistema de Páginas Web, interconectadas y publicadas en Internet, con sitios, lugares, o espacios virtuales, en donde se ubica la información, estos son: identificación, contenidos ,búsquedas ,investigación, evaluación, banco de información horarios, mesa de discusión, asesores (Barbera E. y., S/F)

Como se puede apreciar la ciencia considera al aula virtual como un recurso digital para realizar actividades académicas en procura de conseguir mejoras en la calidad de la educación. Cuando nos referimos a un aula virtual estamos pensando en un conjunto de actividades con un alto ingrediente comunicativo que de otro modo, es decir, sin la existencia del ordenador conectado a la red, no tendrían el sentido que adquiere en el contexto auténtico que proporciona la tecnología.

Por tanto, entendemos que un aula virtual se puede situar dentro de la misma clase mediante una serie de actividades que el maestro puede desarrollar y, aunque podría también funcionar de manera autónoma como sucede en la educación superior, puede cubrir una serie de necesidades educativas nada despreciables realizadas de manera que se libere al profesor y al alumno de la coincidencia temporal e incluso espacial, permitiendo así emprender una educación acorde a los intereses de las nuevas generaciones que prefieren todo aquello que se relaciona con el uso de la tecnología. 
Rev SINAPSIS, Vol. 6, № 1, Junio 2015

La implementación de nuevas tecnologías se ha desarrollado en paralelo con los cambios en los métodos de enseñanza e incluso con la forma de concebir el aprendizaje y la enseñanza, donde cada vez más es el propio alumno quien toma el control del proceso, los materiales y recursos adaptándolos a sus requerimientos y posibilidades.

Los educadores han encontrado un mundo de posibilidades al desarrollo de su práctica docente a través de integrar las nuevas tecnologías como un recurso más en el proceso de enseñanzaaprendizaje, que les ha permitido promover y facilitar la actitud participativa y creadora de los alumnos, la enseñanza individualizada del aprendizaje interactivo, la formación a distancia y de nuevas metodologías como la enseñanza apoyada por computadora, lo que ocasiona una verdadera transformación en el proceso de enseñanza aprendizaje al ceder el papel protagónico al alumno.

El desarrollo de nuevos recursos didácticos y tecnologías educativas ha originado que los docentes que participan en los esfuerzos de formación y capacitación adquieran un mayor protagonismo, intervención y control de los procesos, sobre todo al hacer uso de los recursos y herramientas que mejor se adaptan a sus necesidades formativas. De aquí la importancia de una formación o capacitación planificada, crítica y actualizada (Aguilar, 1997), que tenga como finalidad "incrementar" la calidad de la educación mediante la adquisición de habilidades y conocimientos que permitan a los docentes el desarrollo de actividades pedagógicas creativas, innovadoras y útiles para un desempeño docente eficiente.

El uso de la tecnología en la educación ha sido clave para el desarrollo de la educación a distancia y la creación de tecnologías educativas para el uso cotidiano en las aulas. La implementación de nuevas tecnologías se ha desarrollado en paralelo con los cambios en los métodos de enseñanza e incluso con la forma de concebir el aprendizaje y la enseñanza, donde cada vez más es el propio alumno quien toma el control del proceso, los materiales y recursos adaptándolos a sus requerimientos y posibilidades.

Para tener un aula virtual no es necesaria una tecnología muy complicada. Aunque cuando nos referimos a un aula virtual ésta debería estar tan o más organizada que la clase presencial con todos sus recursos disponibles, para empezar a utilizar el concepto de aula virtual el profesor puede emplear el ordenador de manera que disponga organizadamente de los recursos que le ofrece la red. Entonces, el aula virtual es un entorno de enseñanza - aprendizaje basado en aplicaciones 
Rev SINAPSIS, Vol. 6, № 1, Junio 2015

ISSN $1390-7832$

telemáticas (interacción entre la informática y los sistemas de comunicación), dicho entorno, soporta el aprendizaje colaborativo entre los estudiantes que participan en tiempos y lugares que ellos escojan, mediante una red de computadoras, estableciendo una comunicación entre los alumnos, alumnos y profesores, entre una clase y comunidades académicas o no académicas. (Barbera E. y., S/F)

Mientras avanza la ciencia y la tecnología, la educación debe hacer lo mismo aprovechando los recursos que éstos ofrecen. De acuerdo a la literatura revisada, varios autores coinciden que en un aula virtual se pueden identificar cuatro grandes dimensiones pedagógicas:

Dimensión Informativa. Esta dimensión se refiere al conjunto de recursos, materiales o elementos que presentan información o contenido diverso para el estudio autónomo por parte del alumnado. Sería lo equivalente, por una parte, a los apuntes que el profesor expone en clase que en el contexto del aula virtual pueden adoptar distintos formatos de documentos (texto escrito bien en Word o ODT, bien en formato PDF). Asimismo también pueden incluir el conjunto de recursos o materiales que ayudan a los estudiantes a comprender mejor esos contenidos como son las presentaciones multimedia, las representaciones gráficas, los mapas conceptuales, los videoclips o las animaciones.

Por otra parte, la información o contenido presentada en el aula virtual puede complementarse con otro tipo de archivos, documentos, sitios web o recursos diversos que el profesor selecciona previamente y oferta al alumnado en dicha aula virtual mediante enlaces o hipervínculos. Este conjunto de enlaces hacia otros materiales de Internet permitirán a los estudiantes ampliar el contenido de estudio o acceder autónomamente a nuevos recursos de estudio del curso o asignatura.

La dimensión informativa en un aula virtual, en consecuencia se refiere a todo el conjunto de materiales de distinta naturaleza (textual, multimedia, gráfica, audiovisual) que muestran o ayudan a los estudiantes a acceder autónomamente a los conocimientos objeto de estudio.

Dimensión Práxica: Esta dimensión se refiere al conjunto de acciones, tareas actividades que los estudiantes tienen que realizar en el aula virtual planificadas por el docente para facilitar experiencias de aprendizaje. Estas tareas o actividades pueden ser de diversos tipos:

Participar en foros de debate

La importancia del aula virtual en el bachillerato 
Rev SINAPSIS, Vol. 6, No 1, Junio 2015

ISSN 1390 - 7832

$>$ Leer y redactar ensayos

$>$ Realizar un diario personal

> Plantear y analizar casos prácticos

$>$ Buscar información sobre un tema específico

$>$ Crear una base de datos

$>$ Elaborar proyectos en grupo

$>$ Resolver problemas y/o ejercicios

$>$ Planificar y desarrollar una investigación

$>$ Desarrollar trabajos colaborativos mediante wikis

$>$ Realización de webquests y cazas del tesoro, entre otras

El abanico de posibles tareas o actividades a plantear a los estudiantes en el aula virtual es amplio y lo que se persigue es que éstos desarrollen una experiencia activa en la construcción del conocimiento. Por ello podría indicarse que la dimensión práctica de un aula virtual representa un entorno en el cual el estudiante se enfrenta a situaciones de aprendizaje que implica la activación de distintas habilidades y estrategias tanto cognitivas, actitudinales como sociales. En la medida que el docente realice una adecuada planificación y selección de tareas el modelo de enseñanza implícito en el aula virtual éste tenderá a favorecer un proceso de aprendizaje por recepción (si la mayor parte de las tareas planteadas son de naturaleza repetitiva) o bien un proceso de aprendizaje constructivo (si la mayor parte de las tareas solicitan la búsqueda y análisis activo del conocimiento).

Dimensión Comunicativa: Esta dimensión hace referencia al conjunto de recursos y acciones de interacción social entre estudiantes y el profesor. Esta comunicación se produce a través de herramientas telemáticas tales como los foros, los chats, la mensajería interna, el correo electrónico, la videoconferencia o el audio conferencia. La dimensión comunicativa en un aula virtual es una dimensión sustantiva para la calidad educativa de los procesos de enseñanza-aprendizaje desarrollados a través de e-learning. Si se desconsideran o son poco utilizados estos recursos de comunicación probablemente el aula virtual se convierta en un mero repositorio de documentos y ficheros, pero sin la fluidez y calor humano de una actividad educativa. En este sentido el docente debe propiciar y motivar de modo constante la participación de los estudiantes en los distintos espacios habilitados al efecto en el aula virtual. Normalmente a mayor grado de comunicación entre 
Rev SINAPSIS, Vol. 6, No 1, Junio 2015

ISSN $1390-7832$

los estudiantes y entre éstos y el profesor también se incrementa la motivación, implicación y rendimiento del alumnado en las tareas propuestas.

Dimensión Tutorial y Evaluativa: Esta dimensión hace referencia a las funciones docentes o papel que el profesor debe realizar en el marco de un curso virtual. En la literatura especializada en esta temática se insiste en la figura y papel del tutor a distancia como el elemento clave para el éxito de esta modalidad educativa. En esta línea, la bibliografía coincide en que el docente debe desarrollar más el papel de supervisión y guía del proceso de aprendizaje del alumno que cumplir el rol de transmisor del conocimiento.

Las estrategias metodológicas y el aula virtual

Vivimos en la era del conocimiento y con ésta se generan cambios vertiginosos en todas las esferas. La implementación de nuevas tecnologías se ha desarrollado en paralelo con los cambios en los métodos de enseñanza e incluso con la forma de concebir el aprendizaje y la enseñanza, donde cada vez más es el propio alumno quien toma el control del proceso, los materiales y recursos adaptándolos a sus requerimientos y posibilidades. El desarrollo de nuevos recursos didácticos y tecnologías educativas ha originado que los docentes que participan en los esfuerzos de formación y capacitación adquieran un mayor protagonismo, intervención y control de los procesos, sobre todo al hacer uso de los recursos y herramientas que mejor se adaptan a sus necesidades formativas.

Para utilizar las nuevas tecnologías en educación es preciso integrarlas en la acción didáctica., es decir, es preciso que formen parte del sentido del proyecto de formación. Este hecho que se denomina integración al currículo, se manifiesta como indispensable al pensar en incorporar la computadora en la escuela. (Meza, S/F)

El docente es el diseñador de los procesos metodológicos de cada clase, es el que organiza cada acción, cada recurso a utilizar, siendo necesario que en este diseño involucre a los estudiantes, considerando que cuando los estudiantes participan activamente de este proceso se involucran mucho más en su aprendizaje, con mayor responsabilidad; es decir que los alumnos valoran las intervenciones realizadas por el docente y además los lleva a comprometerse con esta nueva forma de aprender, al parecer es una motivación para involucrarse en su proceso de enseñanzaaprendizaje. (Ferran, 2000) 
Diseñar un entorno de formación supone participar de un conjunto de decisiones a modo de juego de equilibrio entre el modelo pedagógico, los usuarios, según el rol de profesores y alumnos, y las posibilidades de la tecnología desde la perspectiva de la formación flexible (Latona, 1996); (Salinas, 1997b.); (Moran \& Myringer, 1999) sin embargo, muchos de los conceptos asociados con el aprendizaje en la clase tradicional pueden reacomodarse en la utilización de redes para la enseñanza, dando lugar a una nueva configuración formativa que puede superar las deficiencias de los sistemas convencionales, ya sean presenciales o a distancia. De acuerdo a varios estudiosos del tema, lo que frecuentemente se ha procurado es reproducir los modelos de enseñanza-aprendizaje dominantes, y así encontramos muchos cursos y experiencias que se basan fundamentalmente en el modelo clásico de enseñanza-aprendizaje.

Para (Martín-Barbero, 2009), no se inventan nuevas metodologías, sino que la utilización de las TIC en educación abre nuevas perspectivas respecto a una enseñanza mejor, apoyada en entornos en línea cuyas estrategias son prácticas habituales en la enseñanza presencial, pero que ahora son simplemente adaptadas y redescubiertas en su formato virtual. Las posibilidades de las TIC permiten reproducir de alguna forma estos modelos, y en algunos casos puede entenderse que ésta sea la opción «adecuada» (la oportuna combinación de elementos tecnológicos, pedagógicos y organizativos). De ahí la necesidad que el docente actual dentro de sus competencias profesionales desarrolle la que corresponde al uso adecuado de las tecnologías con fines educativos.

El conocimiento, la experiencia, la creatividad y el amor a la educación, son ingredientes de mucha importancia para que los docentes ejecuten variadas estrategias metodológicas; porque actuar estratégicamente supone reflexionar sobre las consecuencias de una u otra opción. De la misma manera dado que las condiciones de actuación donde tiene lugar la toma de decisiones varía durante el transcurso de la acción, el alumno, actor fundamental del proceso educativo, deberá regular constantemente su comportamiento, anticipando esas condiciones y planificando el curso de su actuación, reajustando el proceso y por último, evaluando y corrigiendo los resultados alcanzados en la misma. (Diaz \& González, S/F)

\section{Resultados y discusión}


Rev SINAPSIS, Vol. 6, No 1, Junio 2015

ISSN 1390 - 7832

En la tabla se hace referencia a la frecuencia con que los docentes utilizan el aula virtual para el desarrollo de sus actividades en la enseñanza aprendizaje y que según (Kearsley, 2000), entre los factores importantes en la determinación de una docencia virtual efectiva está el grado de familiaridad que se tenga con la enseñanza virtual. (Anexo 1)

En el cuadro se evidencia que de una población de 16 docentes de bachillerato de la Unidad Educativa ITSUP, las alternativas de todos los días, cada parcial, cada quimestre y nunca reflejaron un $0 \%$; mientras que el total de la población escogieron el segundo literal lo que corresponde al 100\%; indicando así que ellos hacen uso del aula virtual una o dos veces por semana, para el desarrollo de su asignatura.

El aula virtual es un entorno de enseñanza - aprendizaje entre los estudiantes y docentes que establecen una comunicación con el fin de que los estudiantes puedan concebir un buen desempeño académico ya que esta se crea con medios tecnológicos e informáticos logrando que cada alumno sea dueño de su propio conocimiento y lo lleve al destino de la toma de decisiones. Tal y como reflejan los resultados de las encuestas los docentes dieron a conocer que la mejor forma de utilizar el aula virtual es de una o dos veces por semana logrando así mejores resultados académicos.

En el cuadro para conocer el criterio de los docentes sobre el desarrollo de destrezas y habilidades en los estudiantes con el uso del aula virtual, (Barbera E. y., 2005) manifiesta que el estudiante deberá tener las competencias necesarias para gestionar adecuadamente cualquier tipo de comunicación mediada por el ordenador y ponerla al servicio de su aprendizaje, especialmente cuando ésta se produzca de manera asincrónica. (Anexo 2)

De acuerdo a la pregunta realizada a los docentes ¿Está usted de acuerdo que las diferentes actividades en el aula virtual desarrolla en los estudiantes destrezas y habilidades? Se puede evidenciar que $63 \%$ de los docentes eligieron la opción totalmente de acuerdo, de la misma forma el 38\% indicaron la opción de acuerdo, evidenciando que las opciones de desacuerdo y totalmente en desacuerdo, reflejaron un 0\%; quedando así demostrado que usar frecuentemente cada una de las actividades anteriormente mencionadas generan en los alumnos un alto nivel de habilidades y destrezas. 
Rev SINAPSIS, Vol. 6, № 1, Junio 2015

ISSN 1390 - 7832

Las nuevas tecnologías desarrollan en los alumnos habilidades y destrezas, lo cual les permite demostrar desenvolvimiento en sus tareas, el docente debe estar preparado para enfrentar y resolver imprevistos y lograr con el ejemplo todo el proceso de enseñanza aprendizaje, pues la planeación de las propuestas de innovación educativa con apoyo en estas nuevas tecnologías debe considerar, cómo se sitúa el usuario ante la herramienta, qué actividades de aprendizaje realiza, qué valor educativo tienen, qué papel están representado en el proceso de adquisición o elaboración del conocimiento. (Zaldivar, 1993.)

En el cuadro para conocer el criterio de los estudiantes sobre las actividades que realizan en el aula virtual, al respecto (Suárez, 2012) manifiesta que el docente debe determinar las estrategias pedagógicas que se pondrán en marcha, las actividades de aprendizaje y el conjunto de recursos que demandan como una tarea principal de los creadores y tutores de un curso virtual. (Anexo 3)

Como se puede observar claramente en el cuadro con un total de 110 estudiantes, se obtuvieron las siguientes respuestas: videos y foros, $0 \%$; el $6 \%$ eligieron conferencias, demostrando su interés por aprender, de la misma manera optaron por cuestionarios, el 12\%; tomando en cuenta que generan mayor aprendizaje con esta alternativa, mientras que el 6\%; indicaron que utilizan más el chat, mientras que el 48\%; generan mayor interés con las tareas; el 23\%; contestaron que hacen uso de todas las actividades mencionadas en la pregunta, por último el 5\%; mostraron que no participan de ninguna actividad en el aula virtual, quedando reflejado que la gran mayoría de la población estudiantil coincide en que la actividad que más desarrollan son las tareas.

El estudiante no sólo debe conocer cómo navegar en base a las nuevas tecnologías sino que además debe ser el promotor de su propio conocimiento y utilizarlos para potenciar su aprendizaje. El educando deberá tener las competencias necesarias para gestionar adecuadamente cualquier tipo de comunicación mediada por el ordenador y ponerlas al servicio de su aprendizaje (Rodríguez, 2014).

\section{Conclusiones}

Según el análisis efectuado sobre el uso del aula virtual como herramienta de aprendizaje en los estudiantes de bachillerato, se concluyó que tanto los docentes como los estudiantes limitan la utilización del aula virtual en actividades tradicionales como el envío de tareas, evidenciando de 
Rev SINAPSIS, Vol. 6, N 1, Junio 2015

esta manera que se lo considera un recurso más a las actividades modernas de enseñanza aprendizaje y además se desaprovecha la gama de opciones que el aula virtual posee y que despertarían mayor interés en los estudiantes.

\section{Referencias bibliográficas}

1. Aguilar, L. (1997). nternet and the Distance Learning. En el Congreso Internet World 97.

2. Barbera, E. y. (2005). El uso educativo de las aulas virtuales emergentes en la educación superior. Revista de Universidad y Sociedad del conocimiento.

3. Barbera, E. y. (S/F). Hacia el aula virtual: actividades y aprendizaje en la red. Revista Iberoamericana de Educación .

4. Diaz, A., \& González, D. (S/F). La importancia de promover en el aula estrategias de aprendizaje para elevar el nivel académico en los estudiantes. Revista Iberoamericana de Educación (ISSN: 1681-5653) Centro Universitario José Martí Pérez, Cuba.

5. Ferran, M. R. (2000). Estrategias de aprendizaje y motivacion en el desepeñoi academico de los alumnos. Revista Universidad De Barcelona.

6. Gisbert, M. (S/F). El profesor del sigloXXI: de transmisor de contenidos a guia de ciberespacio. Universitat Rovira i Virgili. Tarragona.

7. Harasim, L. (2000). Guía para la enseñanza y el aprendizaje en red. Barcelona.

8. Kearsley, G. (2000). Learning and teaching in cyberspace. Toronto.

9. Latona, K. (1996). Case Studies in Flexible Learning. Sydney: University of Technology/Institute for Interactive Multimedia and Faculty of Education.

10. Martín-Barbero, J. (2009). Cuando la tecnología deja de ser una ayuda didáctica para convertirse en mediación cultural. Revista Electrónica Teoría de la Educación.

11. Meza, A. (S/F). Importancia del Manejo de Estrategias de Aprendizaje para el uso Educativo de . Dirección de soporte educativo.

12. Moran, L., \& Myringer, B. (1999). Flexible learning and university change. Londres.

13. Passey, D. (2000). Education Media International.

14. Rodríguez, A. (2014). Portoviejo.

15. Salinas, J. (1997b.). Enseñanza flexible, aprendizaje abierto. Las redes como herramientas para la formación". 
Rev SINAPSIS, Vol. 6, Nº 1, Junio 2015

16. Suárez, J. I. (2012). Ambiente virtual de aprendizaje como estrategia metodológica para mejorar el rendimiento académico en estudiantes . UNIMINUTO.

17. Zaldivar, A. M.-L. (1993.). Importancia del Manejo de Estrategias de Aprendizaje para el uso Educativo delas Nuevas Tecnologías de Información y Comunicación en Educación. Madrid, Ediciones de la Universidad Complutense.

\section{Anexos}

\section{Anexo 1}

¿Con qué frecuencia utiliza el aula virtual para el desarrollo de sus actividades en la enseñanza aprendizaje?

\begin{tabular}{|c|c|c|c|}
\hline ORDEN & ALTERNATIVAS & F & \% \\
\hline A & Todos los días & 0 & 0,00 \\
\hline B & Una o dos veces por semana & 16 & 100 \\
\hline C & Cada parcial & 0 & 0,00 \\
\hline D & Cada quimestre & Q & 0,00 \\
\hline E & Nunca & 0 & 0,00 \\
\hline & TOTAL & $\mathbf{1 6}$ & $\mathbf{1 0 0 \%}$ \\
\hline
\end{tabular}

Fuente: Docentes de la Unidad Educativa ITSUP

Elaborado por: Las Investigadoras

\section{Anexo 2}

¿Está usted de acuerdo que las diferentes actividades en el aula virtual desarrolla en los estudiantes destrezas y habilidades?

\begin{tabular}{|c|c|c|c|}
\hline ORDEN & ALTERNATIVAS & F & \% \\
\hline A & Totalmente de acuerdo & 10 & 63,00 \\
\hline B & De acuerdo & 6 & 38,00 \\
\hline D & En desacuerdo & 0 & 0,00 \\
\hline E & Totalmente en desacuerdo & 0 & 0,00 \\
\hline & TOTAL & $\mathbf{1 6}$ & $\mathbf{1 0 0 \%}$ \\
\hline
\end{tabular}

Fuente: Docentes de la Unidad Educativa ITSUP

Elaborado por: Las Investigadoras 
Rev SINAPSIS, Vol. 6, No 1, Junio 2015

\section{Anexo 3}

¿Qué actividades desarrolla usted en el aula virtual?

\begin{tabular}{|c|c|c|c|}
\hline ORDEN & ALTERNATIVAS & F & \% \\
\hline A & Videos & 0 & 0,00 \\
\hline B & Conferencias & 7 & 6,00 \\
\hline C & Foros & 0 & 0,00 \\
\hline D & Cuestionarios & 7 & 12,00 \\
\hline E & Chat & 53 & 6,00 \\
\hline F & Tareas & 25 & 23,00 \\
\hline G & Todas las anteriores & 5 & 5,00 \\
\hline H & Ninguna de ellas & $\mathbf{1 1 0}$ & $\mathbf{1 0 0 \%}$ \\
\hline
\end{tabular}

Fuente:

Estudiantes de la Unidad

Educativa ITSUP

Elaborado por: Las

Investigadoras 